\title{
A Rare Case of Polypoidal Cystitis in Adult without History of Prolong Catheterization
}

\author{
Mohd Azren ${ }^{1}$, Mohd Nazli ${ }^{1}$, Asmah Hanim² \\ ${ }^{1}$ Department of Urology, International Islamic University Malaysia Medical Center, \\ Kuantan Pahang \\ ${ }^{2}$ Department of Pathology, International Islamic University Malaysia Medical \\ Center, Kuantan Pahang.
}

Introduction: Polypoidal cystitis is a rare lesion of the bladder mucosa characterized by benign exophytic inflammation,epithelial proliferation and development of polypoid mass without evidence of neoplasm histologically. Polypoid cystitis is rarely seen in patient without prolong used of indwelling catheter. Other cases of polypoidal cystitis are associated with post-radiation, post chemotherapy bladder reaction, colovesicular fistula are extremely rare. Case Report: 66 year old Malay male ,underlying hypertension and Benign Prostatic Hyperplasia (BPH) on treatment, complaining of difficulty in urination, straining on voiding $\hat{A}$ for the past 6 months and on and off $\hat{A}$ hematuria for the past 2 months. He denied any history of CBD insertion or prolong cataheterization. Blood investigations are Normal. X-Ray KUB showed unremarkable. Ultrasound KUB revealed Prostatomegaly, no evidence of renal or ureteric calculi or evidence of Obstructive uropathy, No obvious bladder mass. Cystoscopy Examination (CE) showed enlarged occlusive prostate and noted bladder growth at base of bladder. The growth is malignant looking however no active bleeding seen. No bladder stone seen. Patient subsequently underwent TransUrethral Resection of Bladder Tumor (TURBT) and Trans-Urethral Resection of Prostate (TURP) in same setting. Intra operatively was uneventful. HistoPathological Examination (HPE) from TURBT specimens showed in favour of Polypoidal Cystitis andÂ TURP revealed Nodular Hyperplasia. Discussion: Polypoidal Cystitis is a form or chronic cystitis characterized by exophytic inflammatory $\hat{A}$ lesion of bladder mucosa. The polypoidal mass is mimicking neoplasm. At the initial evaluation, it may be confused with transitional cell carcinoma of the urinary bladder, especially in patient without an indwelling catheter. The most common aetiology of this condition is long-standing indwelling catheterization.. There is alsô̂ reported that any factors that irritates the bladder mucosa may result in Polypoidal Cystitis. $\hat{A}$ In our patient ,there was no history of such other causes, including prolong catheterization, fistula, urinary calculi or radiation therapy. 